\title{
EVALUACIÓN DE CUATRO PATRONES PARA CÍTRICOS INJERTADOS CON LIMA PERSA (Citrus latifolia TAN) BAJO CONDICIONES DE CAÑAS, GUANACASTE
}

\author{
Sergio Hernández ${ }^{1}$
}

\begin{abstract}
RESUMEN
La lima Persa se ha convertido en una alternativa de diversificación agrícola para los agricultores del Distrito Riego Arenal de nuestro país por sus excelentes expectativas de mercado y comportamiento agronómico bajo estas condiciones y también a nivel nacional en general. Esta lima al igual que todas las especies cítricas de importancia comercial en el mundo se propagan principalmente por injerto, lo cual afecta una serie de características fitosanitarias y agronómicas del cultivo que también están condicionadas por el tipo de suelo, clima y aspectos de manejo del árbol. A raíz de ésto, se decidió establecer una parcela bajo condiciones del Pacífico Seco (Estación Experimental Enrique Jiménez Núñez Cañas/ Guanacaste) para evaluar la interacción de cuatro patrones de cítricos: volkameriana ( $\mathrm{Ci}$ trus volkameriana Pasq), taiwanica (Citrus taiwanica), citrumelo [(Poncirus trifoliata (L) Raf $\mathrm{X}$ Citrus paradisi Maca)] y mandarina cleopatra (Citrus reshni Hort $\mathrm{X}$ tan) injertados con un clon de lima Persa seleccionado en la Estación Experimental Enrique Jiménez Núñez libre de enfermedades virosas. Se analizó el rendimiento de fruta fresca, longevidad de árboles, características de crecimiento de árboles, calidad interna y tamaño de fruta y absorción de nutrientes en hoja y fruta en las distintas interacciones lima Persa/patrón. Se encontró que la mayor cantidad de fruta fresca se obtuvo sobre Citrus volkameriana al cabo de tres años de la siembra. Sin embargo, a los cinco años más del 50\% de las plantas de lima Persa injertadas sobre este patrón presentaban decaimiento o habían muerto. Asimismo, la interacción que presentó la mayor eficiencia productiva a los cinco años, fueron los árboles injertados sobre mandarino cleopatra. Los parámetros de calidad interna obtenidos en frutas cosechadas sobre distintos patrones fueron muy similares en tres años de evaluación. El tamaño de fruta sí varió significativamente, encontrándose los mayores calibres y pesos de limas cuando se cosecharon sobre citrumelo. También, se encontraron diferencias significativas en la absorción de nutrientes en hoja y fruta de lima injertada sobre los distintos patrones bajo las condiciones de este estudio.
\end{abstract}

Palabras clave: Patrones, eficiencia productiva, Lima Tahití, Lima bears, Lima mesina.

\section{INTRODUCCIÓN}

La lima Persa se ha convertido en la segunda especie cítrica de importancia comercial en nuestro país después de la naranja por el excelente comportamiento agronómico en nuestras condiciones, grandes expectativas en el mercado nacional e internacional, precocidad en producción y alta rentabilidad.
Elizondo (2004) indica que las exportaciones de lima Persa de Costa Rica para el 2003 mostraron una recuperación importante, se pasó de $8.134 \mathrm{~kg}$ de fruta fresca en el 2002 a $23.412 \mathrm{~kg}$ el año pasado. El precio promedio FOB según el reporte de PROCOMER en el 2003 fue \$ 0,39 por arriba del precio promedio del 2001. En consecuencia, el año anterior se exportó tres veces la cifra del 2002 en volumen y $326 \%$ en valor en el 2003.

\footnotetext{
1 Instituto Nacional de Innovación y Transferencia en Tecnología Agropecuaria (INTA). Costa Rica.
} 
Esta especie cítrica al igual que todas las variedades de interés comercial se propagan por injerto encontrándose en el mundo el uso de distintos portainjertos para su multiplicación mejor adaptados según las características de suelo, clima, tipo de fruta requerida por el mercado o tolerancia a enfermedades.

En general uno de los aspectos de mayor importancia para el éxito en una huerta cítrica es la correcta selección de los patrones a usar, el cual puede influir en tres aspectos fundamentales de la vida productiva del árbol: a) precocidad en producción, b) tolerancia a condiciones adversas del suelo, clima y agentes patogénicos y c) comportamiento frutícola del árbol (Castle 1987).

En la selección de un patrón deben considerase los siguientes aspectos sistemáticamente: a) compatibilidad patrón-injerto b) tolerancia a enfermedades y nematodos, c) limitaciones del sitio y d) características frutícolas. Normalmente se prefieren aquellos patrones de fácil y rápido desarrollo en vivero y plantación, buen enraizamiento, precocidad a la primera cosecha, buena tolerancia a patógenos, porte medio o bajo y buena calidad interna y externa del fruto (Castle1987).

Puede decirse que no existe un patrón que reúna todas las características deseables a la vez, por lo tanto, debe seleccionarse aquel que resuelva los principales problemas existentes en el lugar donde se va a establecer la plantación.

La calidad de la fruta de lima Persa no cambia mucho con el tipo de patrón en contraste con otras especies de cítricos y cultivares, pero si hay grandes diferencias en la producción en campo (Camphell 1979).

Entre los patrones que se recomiendan para la siembra de lima Persa en diferentes regiones del mundo, se encuentran los siguientes:

El Citrus volkameriana es un híbrido de limón, el cual ha mostrado ser muy compatible con la lima Persa formando árboles que producen grandes cosechas precozmente, buen desarrollo de tronco y copa. Es tolerante al virus de la tristeza, xyloporosis y exocortis, pero susceptible a bligth y nematodos de los cí- tricos. Además, se reporta como medianamente tolerante a gomosis (Phytophthora sp.) bajo condiciones normales. Se adapta bien a suelos ligeros, bien drenados y tiene un rango amplio de adaptación al $\mathrm{pH}$, desde suelos ácidos hasta aquellos de $\mathrm{pH}$ alto (Camphell 1991).

Por su parte, la naranja agria (Citrus aurantium L.) fue probablemente hasta $1993 \mathrm{el}$ patrón más ampliamente distribuido en el mundo. Por su problema con el virus de la tristeza cuando es injertado con naranja dulce, pomelo y mandarina decrece de manera importante en Australia, Argentina, Brasil, California, España , Africa del Sur y el Caribe. Este patrón ha demostrado buena adaptación tanto en suelos de textura arenosa, como arcillosa, además crece satisfactoriamente en suelos calcáreos. Es comúnmente usado en suelos pesados y pobremente drenados debido a su moderada tolerancia a Phytophthora. Los árboles injertados sobre este patrón crecen bien sobre suelos con alta salinidad y $\mathrm{pH}$ altos. Es compatible con todas las variedades de limón y limas formando árboles semivigorosos por lo cual se pueden plantar a menor distancia que los árboles injertados sobre Volkameriana. El tamaño de la fruta sobre naranja agria es más pequeña que la obtenida en volkameriana pero más grande que la cosechada en mandarina cleopatra. El contenido de sólidos solubles totales y porcentaje de acidez en el jugo de las frutas producidas sobre este patrón son altos, por ello se ha utilizado mucho en países que producen fruta para mesa. Este patrón desarrolla cierta deformación en la unión de patrón-injerto siendo mayor el diámetro del tronco de la variedad que la del patrón (Castle 1987).

La mandarina cleopatra (Citrus reticulata Blanco) es un patrón que no está ampliamente distribuido en el mundo. Tiene un crecimiento en vivero similar a citrumelo, se comporta bien con las distintas variedades comerciales de naranja, mandarina, pomelo, limones y limas. En Cuba, este patrón injertado con lima Persa tiene una productividad aceptable, con frutos de buen tamaño y calidad con respecto al naranjo agrio. Una de las grandes ventajas de este patrón es su tolerancia a enfermedades virosas como la tristeza, exocortis y xyloporosis. Este patrón es susceptible a los nematodos de los cítricos y 
moderadamente tolerante a la gomosis (Phytophthora). Otra fortaleza es que raramente el blight se presenta antes de 12 a 15 años en árboles cultivados sobre este patrón. La mandarina cleopatra se adapta bien en una amplia variedad de suelos desde ligeros a pesados, aunque las variedades injertadas sobre este patrón son más productivas en suelos ligeramente pesados. Además, tolera bien alta salinidad, $\mathrm{pH}$ elevados y suelos calcáreos. El tamaño de fruta es más pequeña que el obtenido en otros patrones de importancia comercial, sin embargo, la calidad interna es buena en relación con naranja agria. Las especies injertadas sobre cleopatra son poco precoces en producir, pero los árboles son más longevos sobre todo en zonas donde existe tristeza y "Blight" (Camphell1991).

El Citrumelo es un patrón obtenido del cruce naranja trifoliada con grapefruit [(Poncirus trifoliata (L) Raf $\mathrm{X}$ Citrus paradisi Maca)]. El tipo más utilizado en nuestro país ha sido el citrumelo 4475 y la mayor área de siembra de lima Persa se encuentra injertado sobre este patrón, aunque no existen muchas experiencias de este tipo en suelos ácidos (trópico) . Distintos autores no lo recomiendan para limones y limas, lo cual es contradictorio con lo observado bajo nuestras condiciones. Este patrón está adquiriendo gran popularidad en el mundo desde 1974 y hoy día es el más cultivado en la Florida. Las variedades injertadas sobre este patrón tienden a ser más grandes que las obtenidos sobre patrones de mandarina o naranja agria. El Swingle o citrumelo se reporta como tolerante a exocortis, xyloporosis y el virus de tristeza. Es tolerante a unos tipos de nematodos pero susceptible a otros. Es altamente tolerante a gomosis y aparentemente resistente al "Blight", aunque falta información para plantaciones con más de 15 años de edad. El tamaño de la fruta es parecido al obtenido sobre citranges y la calidad del jugo es similar a la encontrada en naranja agria pero más alta que la reportada sobre limones. No se comporta bien en suelos salinos, $\mathrm{pH}$ altos, calcáreos o muy arcillosos (Camphell 1991).

El Taiwanica (C. taiwanica) se obtuvo de una selección de naranja agria y su comportamiento agronómico es similar al de este patrón. Es ligeramente susceptible a gomosis, tanto en suelos arenosos como en suelos pesados. Es de vigor medio y de buena producción, los frutos son bastante ácidos. No tolera los suelos con exceso de sales (Camphell 1991).

En Costa Rica la lima Persa no ha sido evaluada en distintos patrones sistemáticamente y por un tiempo prolongado bajo diferentes localidades.

En consecuencia, el objetivo de este experimento fue evaluar el crecimiento de la lima Persa injertada en diferentes patrones, el estado nutricional de la especie en hoja y fruta, el rendimiento y calidad de fruta, precocidad en producción y longevidad de los árboles en condiciones del Pacífico Seco.

\section{MATERIALES Y MÉTODOS}

El experimento se estableció en la Estación Experimental Enrique Jiménez Núñez del INTA en Cañas, Guanacaste a una altitud de 12 msnm dando inicio en 1993 y terminando en el año 2000. La temperatura media anual osciló entre 25 y $30^{\circ} \mathrm{C}$, la humedad relativa fue del $85 \%$ y la precipitación promedio anual de $1500 \mathrm{~mm}$ durante el período de evaluación. Los árboles se sembraron sobre un suelo Inceptisol, con un $\mathrm{pH}$ de 6,7 y una profundidad superior a los $90 \mathrm{~cm}$, rico en Ca y Mg pero bajo en $\mathrm{P}, \mathrm{Zn}$ y $\mathrm{Mn}$. El contenido de materia orgánica fue de $1,08 \%$ y su textura franco-arenosa con una tabla de agua de 20 $\mathrm{cm}$ durante los meses más lluviosos del año.

Los patrones evaluados en este estudio fueron los siguientes: volkameriana (Citrus volkameriana Pasq.) taiwanica (Citrus taiwanica), citrumelo [(Poncirus trifololiata (L) Raf $\mathrm{X}$ Citrus paradisi Macf )] y mandarina cleopatra (Citrus reshni Hort $\mathrm{X}$ tan).

Se utilizó un diseño de bloques completos al azar con ocho repeticiones, una parcela experimental de cuatro plantas y una útil de dos árboles por tratamiento dejando las dos restantes de borde. Para detectar diferencias entre medias se utilizó pruebas de Medias de Mínimos Cuadrados (LSMEAN). 
En esta parcela se evaluó el crecimiento y producción de la lima en árboles de tres a siete años de edad sembrados a una distancia de $6 \mathrm{~m} \times 5 \mathrm{~m}$, así como el contenido de nutrientes en hoja y fruta, calidad de fruta, precocidad de producción y longevidad de los árboles injertados en los distintos patrones.

Las variables evaluadas para detectar diferencias entre patrones fueron las siguientes: crecimiento: 1) volumen de copa $\left(\mathrm{m}^{3}\right)$ se calculó utilizando la siguiente fórmula $2 / 3 \pi R^{2} H$ para lo cual se midió el radio $(R)$ y altura de copa $(\mathrm{H}), 2$ ) el área foliar contando el número de hojas en un cubo de $0,125 \mathrm{~m}^{3}$ que se colocó en la parte media del árbol en los cuatro puntos cardinales y 3) índice de área foliar relacionando el área foliar y área de proyección de copa. La eficiencia productiva se obtuvo relacionando $\mathrm{kg}$ fruta fresca/ $\mathrm{m}^{2} \mathrm{o} \mathrm{m}^{3}$ de área foliar y la calidad de fruta analizando el contenido de 1) solubles totales, 2) \% acidez, 3) peso y diámetro de fruta ecuatorial y 4) porcentaje de jugo durante tres años en época seca y lluviosa. La longevidad de los árboles se determinó comparando el número de árboles con decaimiento o muertos con respecto a los sembrados en el momento de establecer el experimento (32 árboles en cada patrón). El contenido de nutrientes en el árbol se analizó mediante un estudio de diagnóstico foliar durante tres años en árboles de tres, cuatro y cinco años de edad y análisis de nutrientes en fruta a los cinco y siete años.

\section{RESULTADOS Y DISCUSIÓN}

En este estudio el $C$. volkameriana fue el patrón que produjo la mayor cantidad de frutos de lima Persa de forma más temprana (precocidad). Esto coincide con otros estudios realizados por Passos y Da Cunha en 1981 en Brasil y se atribuye al vigor que inducen los patrones afines al grupo de los limones como volkameriana y macrofila a las variedades comerciales que se injertan sobre ellos.

Se encontró (Cuadro 1) al cuarto año de la siembra, que los árboles de lima Persa injertados sobre $C$. volkameriana tenían una producción superior a las 10 t/ha (318 frutos/árbol) de fruta fresca, mientras que los injer-
Cuadro 1. Valores promedio de rendimiento de fruta fresca anual de lima Persa obtenidos en árboles de distinta edad creciendo sobre diferentes patrones bajo condiciones de $\mathrm{Ca}$ ñas/Guanacaste. 1993 - 2000.*

\begin{tabular}{|c|c|c|c|c|}
\hline $\begin{array}{l}\text { Edad/ } \\
\text { Árbol }\end{array}$ & Patrón & $\begin{array}{c}\text { Frutos/ } \\
\text { Árbol }\end{array}$ & $\begin{array}{c}\text { kg Frutos/ } \\
\text { Árbol }\end{array}$ & $\begin{array}{c}\text { t Fruta/ } \\
\text { ha }\end{array}$ \\
\hline 3 & Cleopatra & 43 & 5,69 & 1,89 \\
\hline 3 & Volkameriana & 82 & 12,14 & 4,03 \\
\hline 3 & Citrumelo & 3 & 0,36 & 0,12 \\
\hline 3 & Taiwanica & 2 & 0,23 & 0,08 \\
\hline 4 & Cleopatra & 104 & 13,74 & 4,57 \\
\hline 4 & Volkameriana & 318 & 43,89 & 14,62 \\
\hline 4 & Citrumelo & 89 & 9,9 & 3,3 \\
\hline 4 & Taiwanica & 26 & 2,75 & 0,92 \\
\hline 5 & Cleopatra & 357 & 48,57 & 16,2 \\
\hline 5 & Volkameriana & 213 & 27,9 & 9,28 \\
\hline 5 & Citrumelo & 15 & 20,12 & 6,7 \\
\hline 5 & Taiwanica & 127 & 14,87 & 4,99 \\
\hline 6 & Cleopatra & 450 & 43,2 & 14,38 \\
\hline 6 & Volkameriana & 555 & 60,5 & 20,13 \\
\hline 6 & Citrumelo & 400 & 46,41 & 15,45 \\
\hline 6 & Taiwanica & 509 & 47,41 & 15,9 \\
\hline 7 & Cleopatra & 337 & 32,35 & 10,78 \\
\hline 7 & Volkameriana & 488 & 53,23 & 17,72 \\
\hline 7 & Citrumelo & 394 & 45,71 & 15,21 \\
\hline 7 & Taiwanica & 462 & 43,4 & 14,4 \\
\hline
\end{tabular}

* Se consideró una densidad de 333 árboles/ha para el cálculo de las toneladas métricas.

tados sobre mandarina cleopatra apenas alcanzaban 4,57 t/ha (104 frutos/árbol). Estas diferencias en producción no fueron significativas de acuerdo a las pruebas de medias empleadas en este experimento.

En el Cuadro 1, también se observa una reducción importante de la producción de los árboles injertados sobre el $C$. volkameriana a partir del quinto año de la siembra lo cual posiblemente se debió a problemas radicales en los árboles muestreados. Estas plantas manifestaron síntomas de decaimiento inducidas posiblemente por asfixia radical (suelos mal drenados) y chancros ocasionados por complejos de hongos; además se sospecha de la presencia de un síndrome transmisible por injerto denominado lime blotch. Los árboles injertados sobre $C$. volkameriana de lima Persa que no decayeron a los seis y siete años produjeron la mayor cantidad de fruta por árbol 
(61 y $53 \mathrm{~kg}$ fruta fresca respectivamente) con respecto al resto de patrones evaluados.

El Cuadro 2, muestra que las plantas de lima Persa injertadas sobre C. volkameriana que sufrieron decaimiento alcanzaban el $41 \%$ al cabo del quinto año y el $53 \%$ a los siete años de edad. Campbell (1991) menciona que este patrón no soporta los suelos con exceso de humedad o mal drenados. Esta característica se presenta con mucha frecuencia en suelos del Pacífico Seco por el tipo de topografía predominante y sus altos contenidos de arcillas. No obstante, Colauto y Viera (2004) informaron que Citrus volkameriana fue uno de los patrones más longevos en estudios realizados con lima Persa en condiciones de Maringa/Brasil. Por tanto, sería conveniente evaluar este patrón que induce características agronómicas muy favorables para la lima en suelos con buenos drenajes, en los cuales las plantas se establezcan sobre lomillos o domos y posean texturas menos pesadas.

En ese mismo cuadro, se observa que a los siete años de edad únicamente el 6,25\% de las limas injertadas sobre citrumelo mani-

Cuadro 2. Porcentaje de árboles de lima Persa injertados en distintos patrones que presentaron problemas de decaimiento y muerte bajo condiciones de Cañas, Guanacaste. 1993 - 2000.

\begin{tabular}{cccrc}
\hline Edad & Cleopatra Volkamericana & \multicolumn{2}{c}{ Citrumelo Taiwanica } \\
\hline 5 & 3,12 & 41 & 0 & 15,6 \\
7 & 12,5 & 53 & 6,25 & 37,6 \\
\hline
\end{tabular}

festaron pérdida de vigor, decaimiento o muerte de árboles. Castle (1998), menciona que citrumelo es un híbrido mejor adaptado a condiciones tropicales, resiste más la asfixia radical y se adapta mejor a suelos con textura pesadas. Hoy día es el patrón más utilizado en el establecimiento de nuevas plantaciones bajo condiciones del Pacífico Seco y Central aunque se reporta como sensible al síndrome del lime blocth (Hernández 2003).

En el Cuadro 3, se presentan los valores promedios de volumen de copa, área foliar y área de proyección de copa encontradas en árboles de lima Persa desarrollados sobre distintos patrones a los tres y cinco años de edad. Nuevamente, las pruebas de medias no detectaron diferencias significativas entre estos árboles de lima bajo estas condiciones. No obstante, las diferencias en volumen de copa a los cinco y siete años de años de edad (Figura 1) entre limas establecidas sobre cleopatra y taiwanica son de 1,31 y $5,56 \mathrm{~m}^{3}$ respectivamente. En el manejo agronómico y cosecha de una plantación estas variaciones de crecimiento son relevantes en los costos de producción, producción/área y tiempo de recuperación de la inversión. A pesar, de las variaciones en área foliar encontradas entre los árboles, los índices de área foliar no fueron significativamente diferentes entre los distintos patrones. En consecuencia, las diferencias en rendimientos de fruta fresca observados en las distintas interacciones parece que no están afectadas por el área foliar que induce cada especie de patrón en cada combinación lima Persa/patrón.

Cuadro 3. Valores promedio de Crecimiento y "Eficiencia Productiva" en $\mathrm{m}^{2} \mathrm{o} \mathrm{m}^{3}$ en árboles de lima Persa de tres y cinco años de edad creciendo sobre diferentes patrones bajo condiciones de Cañas, Guanacaste. 1993 - 2000.

\begin{tabular}{|c|c|c|c|c|c|c|c|}
\hline $\begin{array}{l}\text { Edad/ } \\
\text { árbol }\end{array}$ & Patrón & $\begin{array}{c}\text { Volumen } \\
\qquad\left(\mathrm{m}^{3}\right)\end{array}$ & $\begin{array}{l}\text { Área foliar } \\
\left(\mathrm{m}^{2}\right)\end{array}$ & $\begin{array}{c}\mathrm{kg} / \mathrm{FR} \\
\left(\mathrm{m}^{3}\right)\end{array}$ & $\begin{array}{c}\mathrm{kg} / \mathrm{FR} \\
\left(\mathrm{m}^{2}\right)\end{array}$ & $\begin{array}{c}\text { Área Proyec. } \\
\text { Copa }\left(\mathrm{m}^{2}\right)\end{array}$ & $\begin{array}{l}\text { Índice } \\
\text { área foliar }\end{array}$ \\
\hline 3 & CLEOPAT & 1,74 & 8,28 & 3,13 & 0,66 & 5,27 & 1,61 \\
\hline 3 & VOLKAM & 2,33 & 10,65 & 5,36 & 1,18 & 7,62 & 1,47 \\
\hline 3 & CITRUM & 1,62 & 7,5 & 0,25 & 0,05 & 4,85 & 1,59 \\
\hline 3 & TAIWAN & 3,05 & 11,92 & 0,07 & 0,02 & 7,56 & 1,65 \\
\hline 5 & CLEOPAT & 5,7 & 18,95 & 8,72 & 2,6 & 18,47 & 1,03 \\
\hline 5 & VOLKAM & 9,62 & 27,7 & 2,96 & 1,06 & 28,5 & 1,00 \\
\hline 5 & CITRUM & 6,81 & 22,15 & 2,94 & 0,91 & 19,07 & 1,17 \\
\hline 5 & TAIWAN & 11,26 & 24,9 & 1,3 & 0,62 & 26,2 & 0,94 \\
\hline
\end{tabular}




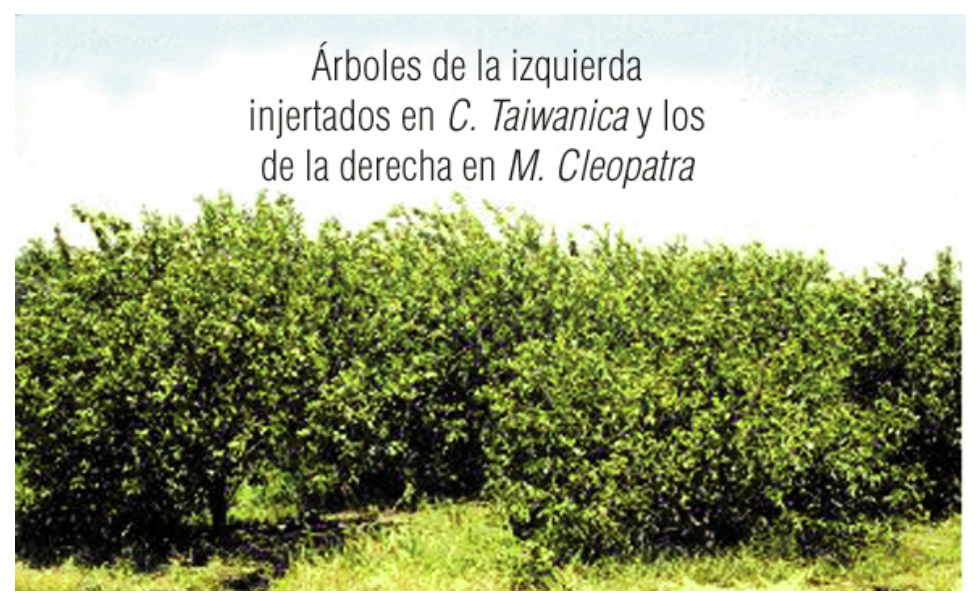

Figura 1. Crecimiento de árboles de lima Persa con cinco años de edad injertados en dos patrones de cítricos bajo condiciones de Cañas/Guanacaste. 1993 - 2000.

Los mayores índices de eficiencia productiva $\left(\mathrm{kg} / \mathrm{m}^{3}\right)$ en árboles de tres años de edad se encontraron en limas injertadas sobre volkameriana y mandarino cleopatra. En el quinto año este parámetro se redujo en las plantas establecidas sobre volkameriana debido a los factores mencionados y al área de proyección de copa que adquieren las limas cuando crecen sobre este patrón. Los árboles que presentaron los mayores índices de eficiencia productiva al quinto año, fueron los injertados sobre cleopatra.

En el Cuadro 4, se presentan las características organolépticas de fruta de tres meses de edad después de las antesis obtenidas sobre distintos patrones bajo condiciones de Cañas/Guanacaste. Esta valoración se realizó en frutos cosechados en época seca y lluviosa en árboles con cinco años de edad. Los datos demuestran que existen pocas diferen- cias en cuanto a calidad interna de fruta (Brix ${ }^{\circ}, \%$ ácido, ratio y $\mathrm{pH}$ ) lo cual coincide con lo indicado por Camphell (1979) en estudios realizados en la Florida. Sin embargo, bajo nuestras condiciones se han detectado importantes diferencias en el contenido de jugo en frutos cítricos obtenidos sobre distintos patrones, encontrándose en algunos casos que la fruta cosechada sobre patrones muy vigorosos como el volkameriana, presenta un excesivo crecimiento con bajo contenido de jugo especialmente en las primeras cosechas del árbol e independientemente de la región.

En cuanto a tamaño se observa que los frutos cosechados sobre citrumelo presentaron valores de peso y diámetro ecuatorial significativamente superiores al resto de la fruta cosechada sobre otros patrones. Es importante resaltar que el tamaño de fruta obtenido sobre mandarina cleopatra no fue diferente al

Cuadro 4. Características de calidad interna de frutos de lima Persa obtenidos sobre distintos patrones bajo condiciones de Cañas, Guanacaste. 1993 - 2000.

\begin{tabular}{lccccccc}
\hline Patrón & $\begin{array}{c}\text { Peso } \\
(\mathbf{g})\end{array}$ & $\begin{array}{c}\text { Diam. } \\
\text { Ecuat. (cm) }\end{array}$ & BRIXo & $\begin{array}{c}\text { \% } \\
\text { Ácido }\end{array}$ & $\begin{array}{c}\text { Relac. } \\
\text { (Brix/Ácido) }\end{array}$ & pH & $\begin{array}{c}\% \\
\text { Jugo* }\end{array}$ \\
\hline CLEOP & $82,87 \mathrm{c}$ & $5,1 \mathrm{~b}$ & 7,98 & 8,25 & 0,98 & 2,88 & $38,8 \mathrm{a}$ \\
VOLKAM & $86,6 \mathrm{bc}$ & $5,2 \mathrm{~b}$ & 8,2 & 7,76 & 1,04 & 2,67 & $35,16 \mathrm{~b}$ \\
CITRUM & $95,95 \mathrm{a}$ & $5,4 \mathrm{a}$ & 7,98 & 7,73 & 1,10 & 2,68 & $35,3 \mathrm{~b}$ \\
TAIWAN & $76,5 \mathrm{c}$ & $4,9 \mathrm{c}$ & 7,55 & 8,68 & 0,9 & 2,67 & $34,5 \mathrm{~b}$ \\
\hline
\end{tabular}

*Valores en columna con la misma letra no difieren significativamente entre sí (5\%). 
observado sobre volkameriana y taiwanica lo cual contradice las observaciones de Campbell (1991) que menciona que la fruta de lima Persa cosechada sobre cleopatra es muy pequeña. Esto posiblemente se debe a que existe muy poca información sobre el comportamiento de la lima Persa injertada sobre mandarina cleopatra creciendo bajo condiciones tropicales. Asimismo, los frutos cosechados sobre mandarino cleopatra presentaron diferencias significativas en porcentaje de jugo con respecto a las limas cosechadas en otros patrones. En general el porcentaje de jugo fue ligeramente inferior a lo establecido por la Norma Codex que menciona un $42 \%$ como mínimo, aunque esto puede variar según el destino del producto.

En el Cuadro 5, se observa que los contenidos foliares de macro y micro nutrientes en árboles de lima Persa injertados sobre distintos patrones presentaron importantes variaciones entre años de muestreo para un mismo patrón cuando se realizaron análisis en época seca y húmeda. Esto podría deberse a la edad del árbol y al rendimiento en producción, al manejo agronómico de la plantación y al programa de fertilización sugerido

Cuadro 5. Diagnóstico foliar de árboles de lima Persa en diferentes edades creciendo sobre distintos patrones bajo condiciones de Cañas, Guanacaste. 1993 - 2000.

\begin{tabular}{|c|c|c|c|c|c|}
\hline Nutriente & $\begin{array}{c}\text { Edad/árbol } \\
\text { años }\end{array}$ & Cleopatra* & Volkamericana* & Citrumelo* & Taiwanica* \\
\hline $\mathrm{N}$ & 3 & 1,89 & 1,9 & 2,0 & 1,89 \\
\hline \multirow[t]{2}{*}{$(\%)$} & 4 & 2,06 & 1,95 & 1,89 & 1,88 \\
\hline & 5 & 2,61 & 2,6 & 2,6 & 2,5 \\
\hline$P$ & 3 & 0,22 & 0,22 & 0,26 & 0,26 \\
\hline \multirow[t]{2}{*}{$(\%)$} & 4 & $0,13 b$ & $0,13 b$ & $0,15 b$ & $0,23 \mathrm{a}$ \\
\hline & 5 & 0,17 & 0,18 & 0,21 & 0,21 \\
\hline $\mathrm{K}$ & 3 & $1,24 b$ & $1,54 \mathrm{a}$ & $1,48 \mathrm{a}$ & $1,5 \mathrm{a}$ \\
\hline \multirow{2}{*}{$(\%)$} & 4 & $1,20 \mathrm{~b}$ & $1,59 b$ & $1,43 \mathrm{a}$ & $1,68 \mathrm{a}$ \\
\hline & 5 & $1,33 \mathrm{c}$ & $1,81 \mathrm{a}$ & $1,6 \mathrm{a}$ & $1,97 \mathrm{a}$ \\
\hline $\mathrm{Ca}$ & 3 & $1,42 b$ & $1,48 b$ & $1,42 b$ & $1,61 \mathrm{a}$ \\
\hline \multirow[t]{2}{*}{$(\%)$} & 4 & $1,31 \mathrm{~b}$ & $1,25 b$ & $1,27 b$ & $1,43 \mathrm{a}$ \\
\hline & 5 & $1,34 \mathrm{~b}$ & $1,38 b$ & $1,32 \mathrm{a}$ & $1,43 a$ \\
\hline $\mathrm{Mg}$ & 3 & $0,27 \mathrm{a}$ & $0,18 \mathrm{c}$ & $0,22 b$ & $0,23 b$ \\
\hline \multirow[t]{2}{*}{$(\%)$} & 4 & $0,35 \mathrm{a}$ & $0,21 c$ & $0,29 b$ & $0,30 \mathrm{~b}$ \\
\hline & 5 & $0,34 \mathrm{a}$ & $0,25 b$ & 0,33 a & $0,32 \mathrm{a}$ \\
\hline $\mathrm{Cu}$ & 3 & $2,75 b$ & $3,0 \mathrm{a}$ & $3,62 \mathrm{a}$ & $3,25 \mathrm{a}$ \\
\hline \multirow[t]{2}{*}{ ppm } & 4 & $2,5 \mathrm{~b}$ & $3,25 \mathrm{a}$ & $3,5 \mathrm{a}$ & $2,87 \mathrm{a}$ \\
\hline & 5 & $2,62 \mathrm{c}$ & $3,62 a b$ & 4,25 a & 3,12 bc \\
\hline $\mathrm{Zn}$ & 3 & $16,1 \mathrm{a}$ & $16,4 a b$ & $13,2 b$ & $16,9 \mathrm{a}$ \\
\hline \multirow[t]{2}{*}{ ppm } & 4 & 15,1 & 16,2 & 14,12 & 16,9 \\
\hline & 5 & $40 \mathrm{bc}$ & $42 a b$ & $38,9 \mathrm{c}$ & $43 c$ \\
\hline $\mathrm{Mn}$ & 3 & $20,87 a$ & $20,0 \mathrm{a}$ & $15,37 \mathrm{~b}$ & $23,12 \mathrm{a}$ \\
\hline \multirow[t]{2}{*}{ ppm } & 4 & 28,0 a & 28,0 a & 20,62 b & 30,5 a \\
\hline & 5 & $21,6 \mathrm{bc}$ & $26,5 \mathrm{a}$ & $19,5 \mathrm{c}$ & $24,4 a b$ \\
\hline $\mathrm{Fe}$ & 3 & 90,8 & 99,2 & 104,9 & 98,2 \\
\hline \multirow[t]{2}{*}{ ppm } & 4 & 194,1 b & 2045 b & 248,8 a & 187,6 b \\
\hline & 5 & $110,8 b$ & $133 \mathrm{~b}$ & $166,1 \mathrm{a}$ & $109,1 \mathrm{~b}$ \\
\hline
\end{tabular}

*Valores en columna con la misma letra no difieren significativamente entre sí (5\%). 
en cada año considerando cantidades de fertilizantes, épocas, forma y frecuencia de aplicación. Asimismo, en el Cuadro 5, se aprecian importantes diferencias significativas en las concentraciones de algunos nutrientes entre los árboles de lima desarrollados sobre los distintos patrones en el mismo año. Wutscher (1988) al respecto menciona que los patrones tienen distinta capacidad de absorción de nutrientes y agua, lo cual afecta dentro de la planta aspectos generales del árbol como el crecimiento y de la fruta como tamaño y calidad. En general se observa, que los contenidos foliares de la mayoría de nutrientes en los distintos años de muestreo son más altos en los patrones más vigorosos como volkameriana y taiwanica. En cambio las concentraciones de $\mathrm{K}$ en las hojas de las limas injertadas sobre mandarina cleopatra fueron significativamente inferiores a las encontradas en los otros patrones prácticamente durante los tres años de muestreo.

La concentración de calcio era alta en el suelo sobre el cual se estableció este estudio, sin embargo, durante el período de evaluación los contenidos en hoja fueron inferiores al contenido normal de acuerdo a tablas de la Florida para todas las plantas independientemente del patrón.

Las plantas injertadas sobre citrumelo presentaron los menores contenidos de $\mathrm{Zn}$ en las hojas, lo cual coincide con estudios realizados por otros investigadores en la Florida
(Camphell 1979). También, en citrumelo se detectaron los mayores contenidos de hierro y las menores concentraciones de manganeso durante los tres años de muestreo. En otros patrones de cítricos como Carrizo bajo condiciones de la Huetar Norte esta baja relación que se establece entre el contenido de Fe y $\mathrm{Mn}$ induce un síndrome conocido como raíz corchosa.

Finalmente, en el Cuadro 6, se presentan para cada interacción evaluada las cantidades de macronutrientes en gramos/tonelada de fruta fresca que fueron removidos en dos cosechas cuando los árboles tenían cinco y siete años de edad. Para ello, se analizó fruta cosechada en época seca y lluviosa encontrándose que las cantidades de fósforo y potasio fueron significativamente inferiores cuando las limas se obtuvieron de árboles injertados sobre mandarina cleopatra. Este resultado es similar al encontrado en hoja para este patrón donde también se detectaron las menores concentraciones de éstos y otros nutrientes. Por tanto, pareciera que la mandarina cleopatra es un patrón poco extractor de nutrientes pero productivo tal y como se observa (Cuadro 1) al compararlo con las otras interacciones bajo estas condiciones de experimento. En consecuencia, este resultado podría tener importantes implicaciones en los programas de fertilización y las cantidades de nutrientes aplicadas anualmente de acuerdo a las toneladas de fruta fresca cosechada sobre distintos patrones.

Cuadro 6. Nutrientes removidos en gramos/tonelada de fruta fresca de lima Persa en distintos patrones de cítricos, bajo condiciones de Cañas, Guanacaste. 1993 - 2000.*

\begin{tabular}{cccccc}
\hline Nutriente & Edad/árbol & Cleopatra $^{*}$ & Volkamericana $^{*}$ & Citrumelo* $^{*}$ & Taiwanica* $^{*}$ \\
\hline \multirow{2}{*}{$\mathrm{N}$} & 5 & $1550 \mathrm{~b}$ & $1800 \mathrm{a}$ & $1580 \mathrm{~b}$ & $1620 \mathrm{~b}$ \\
& 7 & 1130 & 1090 & 1250 & 1190 \\
$\mathrm{P}$ & 5 & $160 \mathrm{~b}$ & $170 \mathrm{ab}$ & $180 \mathrm{a}$ & $180 \mathrm{a}$ \\
& 7 & $200 \mathrm{~b}$ & $210 \mathrm{ab}$ & $230 \mathrm{a}$ & $270 \mathrm{a}$ \\
$\mathrm{K}$ & 5 & $1860 \mathrm{~b}$ & $2140 \mathrm{a}$ & $2130 \mathrm{a}$ & $2240 \mathrm{a}$ \\
& 7 & $1650 \mathrm{~b}$ & $1980 \mathrm{a}$ & $1850 \mathrm{ab}$ & $1910 \mathrm{a}$ \\
$\mathrm{Ca}$ & 5 & 590 & 560 & 520 & 600 \\
& 7 & 370 & 350 & 400 & 390 \\
$\mathrm{Mg}$ & 5 & 120 & 110 & 130 & 110 \\
& 7 & 100 & 110 & 110 & 110 \\
\hline
\end{tabular}

*Valores en columna con la misma letra no difieren significativamente entre sí (5\%). 


\section{CONCLUSIONES}

La mayor precocidad en producción y volumen de copa se obtuvo cuando se injertó la lima Persa sobre el Citrus volkameriana. Asimismo, a los cinco años los árboles de lima Persa que presentaron el menor volumen de copa fueron los injertados sobre mandarina cleopatra.

Los mayores índices de producción en kg de fruta fresca $/ \mathrm{m}^{3} \mathrm{o} / \mathrm{m}^{2}$ de copa se alcanzaron cuando la lima Persa se injertó sobre $C$. volkameriana y mandarina Cleopatra.

Los porcentajes más bajos de árboles muertos o con decaimiento de lima Persa se presentaron en las interacciones con citrumelo y mandarina cleopatra a los siete años de establecida la plantación.

Para fines industriales los frutos de lima Persa que presentaron la mayor cantidad de jugo fueron los injertados sobre mandarina cleopatra.

En el establecimiento de una plantación comercial de lima Persa deberían considerarse las bondades agronómicas y sanitarias que ofrecen los distintos patrones de cítricos evaluados en este experimento.

\section{AGRADECIMIENTO}

Se desea expresar el más sincero agradecimiento a la Tec. Sara Rodríguez Hernández funcionaria del Instituto Nacional de Innovación y Transferencia en Tecnología Agropecuaria (INTA) con sede en la Estación Experimental Enrique Jiménez Núñez por su decidida colaboración en la parte de campo de este experimento.

\section{LITERATURA CITADA}

Campbell,C. W. 1979. Tahiti lime production in Florida. University of Florida. Florida Cooperative Service. EUA. p. 102.

Camphell, C, W. 1991. Rootstocks for Tahiti lime. Proc. Fla. State Hort. Soc. 104: 28-30.

Castle, W. S. 1987. Citrus rootstocks. In: Rom, R.C. and Carlson, R.F. eds. Rootstocks for Fruit Crops. John Wiley and Sons, New Yok, p. 361-399.

1998. Rootstcks reflections. Citrus Review FI 79 (9): 11-16.

Coloauto,S.N.; Viera, J.N.C. 2004. Rootstocks for Tahití lime.Scientia Agrícola 61(2):1-7.

Elizondo, A. 2004. Situación actual y perspectivas del mercado. En curso sobre manejo, producción y comercialización de la lima Persa (Citrus latifolia Tan). Memoria. Puntarenas, Costa Rica. p. 1-10.

Hernández, S. 2003. Evaluación del crecimiento, productividad, calidad de fruta y contenido nutriconal en hoja y fruto de lima Persa ( $\mathrm{Ci}$ trus latifolia Tan) injertada en cuatro patrones de cítricos bajo condiciones de Cañas/ Guanacaste. En Día de Campo lima Persa Guanacaste, Costa Rica. p. 45.

Passos O. S.; A. P. Da Cunha Sobrinho 1981. Citrus Roostocks in Brazil. Proc. Int. Soc. Citriculture. 102-105.

Wutscher, H.K. 1988. Rootstock effects on fruit quality. In factors Affecting Fruit Quality. Lake Alfred. Florida. p. 24. 